\title{
Interfacial tension of electrolyte solutions
}

Cite as: J. Chem. Phys. 113, 9722 (2000); https://doi.org/10.1063/1.1321043

Submitted: 02 May 2000 . Accepted: 06 September 2000 . Published Online: 16 November 2000

Yan Levin

\section{ARTICLES YOU MAY BE INTERESTED IN}

The Surface Tension of Debye-Hückel Electrolytes

The Journal of Chemical Physics 2, 528 (1934); https://doi.org/10.1063/1.1749522

Surface tension of electrolyte interfaces: Ionic specificity within a field-theory approach The Journal of Chemical Physics 142, 044702 (2015); https://doi.org/10.1063/1.4905954

Salt effects on the surface tensions of dilute electrolyte solutions: The influence of nonzero relative solubility of the salt between the coexisting phases

The Journal of Chemical Physics 80, 6225 (1984); https://doi.org/10.1063/1.446725

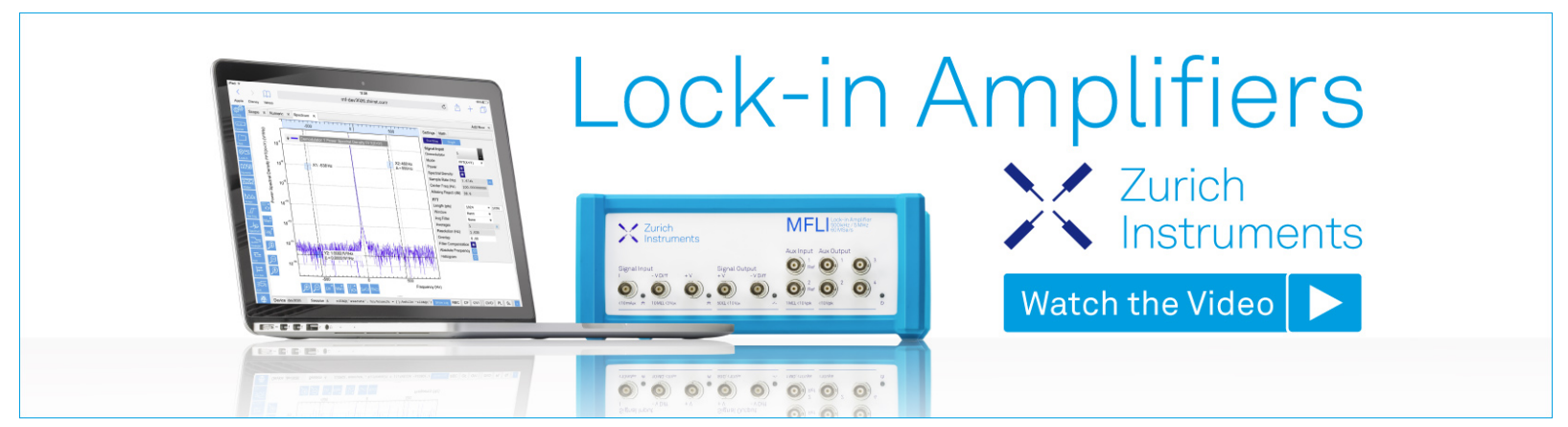




\title{
Interfacial tension of electrolyte solutions
}

\author{
Yan Levin ${ }^{\mathrm{a})}$ \\ Instituto de Física, Universidade Federal do Rio Grande do Sul Caixa Postal 15051, CEP 91501-970, \\ Porto Alegre, RS, Brazil
}

(Received 2 May 2000; accepted 6 September 2000)

\begin{abstract}
A theory is presented to account for the increase in surface tension of water in the presence of electrolyte. Unlike the original "grand-canonical" calculation of Onsager and Samaras, which relied on the Gibbs adsorption isotherm and led to a result which could only be expressed as an infinite series, our approach is "canonical" and produces an analytic formula for the excess surface tension. For small concentrations of electrolyte, our result reduces to the Onsager-Samaras limiting law. () 2000 American Institute of Physics. [S0021-9606(00)50345-6]
\end{abstract}

\section{INTRODUCTION}

It has been known for almost a century that aqueous solutions of inorganic salts have greater surface tension than pure water. ${ }^{1}$ An explanation of this effect was advanced by Wagner $^{2}$ in 1924 on the the basis of the theory of strong electrolytes, which was introduced only a year earlier by Debye and Hückel. ${ }^{3}$ The fundamental insight of Wagner was to realize that the presence of ions polarizes the air-water interface, inducing an effective surface charge. Since the dielectric constant of water is significantly larger than that of air, each ion's image charge equals it in sign and magnitude. Thus, the repulsive interaction with the images reduces the density of electrolyte near the interface. Appealing to the Gibbs adsorption isotherm, ${ }^{4}$ it is evident that the depletion of solute near the interface results in an increase of surface tension. Furthermore, the experimental observation that for small concentrations this increase depends only weakly on the ionic size ${ }^{1,5-8}$ suggests the existence of a universal limiting law, similar to the one obtained by Debye and Hückel for bulk properties of electrolyte solutions. ${ }^{3}$ Indeed, the calculation of Onsager and Samaras (OS) seems to confirm such a limiting law. ${ }^{9}$ A number of approximations adopted by OS in the course of calculations, however, obscure the full range of validity of their findings.

To check the thermodynamic consistency of the OS results it is, therefore, worthwhile to explore other routes to surface tension. In the absence of an exact calculation, these will provide a way to assess the self-consistency of the formulas obtained. To this end, we propose a new approach for calculating the increase in interfacial tension of water due to 1:1 electrolyte. Our method differs from that of OS-who integrated the Gibbs adsorption isotherm-in that we identify the excess surface tension directly with the Helmholtz free energy necessary to create an interface. The advantage of this approach is that it allows the writing of an analytic formula for the excess surface tension. This should be contrasted with the method of OS, who were able to express their result only as an infinite sum. Nevertheless, in the limit of large dilution our expression reduces to that of OS, sug-

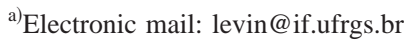

gesting that the limiting law is, indeed, exact. The paper is organized as follows: in Sec. II we briefly review the thermodynamics of interfaces and the relationship between the canonical and the grand-canonical routes to surface tension; in Sec. III we outline the OS theory and present our calculations; the results and conclusions are summarized in Sec. IV.

\section{THERMODYNAMICS OF INTERFACES}

Consider an $r$ component mixture confined to volume $V$. The system is in contact with a hypothetical reservoir of solute particles at temperature $T$ and chemical potential $\mu_{i}$, with $\{i=1, \ldots, r\}$. If the periodic boundary conditions are imposed on $V$, at equilibrium, the system will have $\left\{N_{i}\right\}$ particles uniformly distributed throughout the volume.

On the other hand, if $V$ forms part of a larger system, its domain must be delimited by an interface. We shall idealize this interface as a mathematical surface-the Gibbs dividing surface for solvent. The discontinuity produced by the interface will affect the interactions between the particles in its vicinity. If the interface-particle interaction is repulsive, it will lead to the depletion of solute from the interfacial region, forcing it back into reservoir. On the other hand, if this interaction is attractive, a concentrated layer of solute will build up along the interface, producing a net flow of solute from the reservoir. When the equilibrium is reestablished, the distribution of solute is no longer uniform, but characterized by a density profile $\rho_{i}(z)$, where $z$ is a distance from the interface. Clearly, if the system has a thermodynamic limit, the interface does not influence the bulk distribution of particles and $\rho_{i}(\infty)=\rho_{i}$, where $\rho_{i}=N_{i} / V$. The presence of an interacting interface, however, is responsible for a net increase or decrease of solute in the system. Thus, we define the amount of solute "adsorbed" as

$$
N_{i}^{s}=\int_{0}^{\infty}\left(\rho_{i}(z)-\rho_{i}\right) d z .
$$

Note that this quantity can be either positive or negative depending on whether the solute enters or leaves the system. 
Now, from general thermodynamic principles, the change in the total Helmholtz free energy of a system at fixed volume, temperature, and the amount of solvent, is

$$
d F=\sigma d A+\sum_{i=1}^{r} \mu_{i} d N_{i},
$$

where $\sigma$ is the surface tension and $A$ is the area of the interface. We shall now divide the solute particles into the "bulk" $N_{i}^{b}$ and the "surface" $N_{i}^{s}$. Equation (2) can also be separated into the bulk

$$
d F^{b}=\sum_{i=1}^{r} \mu_{i} d N_{i}^{b},
$$

and the surface contribution

$$
d F^{s}=\sigma d A+\sum_{i=1}^{r} \mu_{i} d N_{i}^{s} .
$$

Since $d F^{s}$ is an extensive function of $A$ and $\left\{N_{i}^{s}\right\}$, the Euler's theorem for first-order homogeneous functions allows us to integrate Eq. (4), yielding

$$
F^{s}=\sigma A+\sum_{i=1}^{r} \mu_{i} N_{i}^{s} .
$$

On the other hand, differentiating Eq. (5) and comparing it with Eq. (4), we find a Gibbs-Duhem-type equation

$$
A d \sigma+\sum_{i=1}^{r} N_{i}^{s} d \mu_{i}=0 .
$$

For $r=1$, this reduces to the Gibbs adsorption isotherm

$$
\left.\frac{\partial \sigma}{\partial \mu}\right|_{T, V}=-\frac{N^{s}}{A} .
$$

Since the thermodynamic stability requires $d \mu / d \rho>0$, where $\rho$ is the concentration of solute, it is evident that a positive adsorption leads to a decrease in surface tension, while a negative adsorption increases the surface tension. This, then, explains Wagner's original observation that the repulsion of ions from a polarized air-water interface results in a depletion of electrolyte and an increase in surface tension.

Knowledge of $N_{i}^{s}$ allows a calculation of the excess surface tension through the integration of Eq. (6). Following Wagner, this was the procedure adopted by OS.

The discussion outlined above relies on the presence of a hypothetical reservoir. In the language of statistical mechanics it is intrinsically grand canonical. A different, canonical calculation should also be possible. In the thermodynamic limit, the choice of ensemble will not matter, if an exact calculation is performed. In practice, however, no exact calculation is possible and approximations have to be made. Thus, there is no a priori guarantee that the two ensembles will lead to identical results. The canonical approach, presented below, is conceptually simpler than its grandcanonical counterpart, since no reservoir is present. Thus, when the interactions between the interface and the solute are turned on, the particles do not leave the systems and $N_{i}^{s}$ $=0$. Therefore, canonically the interfacial tension is equiva- lent to the surface Helmholtz free-energy density, $\sigma$ $=F^{s} / A$. Now, consider a mixture confined to a cylinder of length $H$ and a cross-sectional area $A$. Define $F$ as the total Helmholtz free energy of solute and $F^{\text {bulk }}$ as the free energy of solute in the absence of an interface. The change in the surface tension of solvent due to addition of solute is then

$$
\sigma^{e x}=\lim _{A \rightarrow \infty} \frac{1}{A} \lim _{H \rightarrow \infty}\left(F-F^{\text {bulk }}\right) .
$$

\section{THE SURFACE TENSION}

We are interested in the surface tension of an interface between an aqueous solution of a symmetric 1:1 electrolyte and air. The extension to asymmetric electrolytes is, in principle, straightforward. Some extra care, however, has to be taken to account for the strong correlations between the cations and the anions, which result from an increased ionic charge. ${ }^{10,11}$ In view of the experimental observation that for small concentrations of electrolyte the excess surface tension depends only weakly on the ionic size, ${ }^{1,7,8}$ to simplify the calculations we shall treat ions as point-like. The solvent will be modeled as a uniform dielectric medium.

According to statistical mechanics, the concentration of solute a distance $z$ from the interface is given by the Boltzmann distribution

$$
\rho_{i}(z)=\rho_{i} e^{-\beta W_{i}(z)},
$$

where $\beta=1 / k_{B} T$, and $W_{i}(z)$ is the adsorption potential of the species $i$ and is defined as the work required to bring a particle from infinity to distance $z$ from the interface. For symmetric electrolyte $W_{+}(z)=W_{-}(z) \equiv W(z)$, and $\rho_{+}(z)$ $=\rho_{-}(z) \equiv \rho(z)$. Now, consider an ion located at distance $z$ from the interface. If the electrolyte is infinitely dilute, the electrostatic potential distance $r_{1}$ from the ion can be calculated directly from the Laplace equation

$$
\psi\left(r_{1}, r_{2}\right)=\frac{q}{D r_{1}}+\frac{\left(D-D^{\prime}\right)}{\left(D+D^{\prime}\right)} \frac{q}{D r_{2}},
$$

where $D$ is the dielectric constant of water, $D^{\prime}$ is the dielectric constant of air, and $r_{2}$ is the distance from the image charge located opposite the ion at $-z$. From the second term of Eq. (10), the charge of the "image ion" is $q_{\text {image }}=(D$ $\left.-D^{\prime}\right) q /\left(D+D^{\prime}\right)$. For an aqueous solution close to room temperature $D \approx 80$ and $D^{\prime} \approx 1$, so that $q_{\text {image }} \approx q$. Therefore, in a perturbative theory, $D^{\prime} / D$ can play the role of a small parameter. Since $D \gg D^{\prime}$, the zeroth-order calculation is already quite accurate, and $D^{\prime}$ can be set to zero. This is the first approximation proposed by Wagner ${ }^{2}$ and used by OS. ${ }^{9}$ In the following discussion we shall also adopt this approximation. The repulsive force felt by an ion due to the dielectric discontinuity produced by an air-water interface is then

$$
\mathcal{F}(z)=\frac{q^{2}}{4 D z^{2}} .
$$

The amount of work required to bring this ion from infinity to a distance $z$ from the interface is 


$$
W_{\infty}(z)=-\int_{\infty}^{z} \mathcal{F}(x) d x=\frac{q^{2}}{4 D z},
$$

where we have added the subscript $\infty$ to $W(z)$ to emphasize that the calculation is done at infinite dilution. Alternatively, the Güntelberg charging process ${ }^{12}$ can be used to calculate the amount of electrostatic work necessary to "create" an ion of charge $q$ at distance $z$ from the interface

$$
W_{\infty}(z)=\int_{0}^{1} \frac{\lambda q}{2 D z} q d \lambda=\frac{q^{2}}{4 D z} .
$$

If the electrolyte is at finite concentration, the electrostatic potential in the vicinity of a fixed ion satisfies the Debye-Hückel equation

$$
\nabla^{2} \psi=\kappa^{2} \psi
$$

where $\kappa^{2}(z)=8 \pi \rho(z) / D k_{B} T$. In order to simplify the calculations, Onsager and Samaras suggested replacing $\kappa(z)$ in Eq. (14) by its bulk value $\kappa(\infty) .{ }^{9}$ This certainly seems like a reasonable thing to do, in view of the fact that for an aqueous solution at room temperature the boundary layer is very narrow and the density profile rapidly approaches its bulk value. Nevertheless, this approximation introduces some internal inconsistency into the theory which can, in principle, manifest itself when different thermodynamic routes are taken to calculate the excess surface tension. To zeroth order in $D^{\prime} / D$ and with $\kappa(z) \rightarrow \kappa(\infty)$, Eq. (14) can be integrated, ${ }^{9,13}$ yielding

$$
\psi\left(r_{1}, r_{2}\right)=\frac{q e^{-\kappa r_{1}}}{D r_{1}}+\frac{q e^{-\kappa r_{2}}}{D r_{2}},
$$

where, once again, $r_{1}$ is the distance from the ion, and $r_{2}$ is the distance from the image charge located at $-z$. The adsorption potential is obtained through the Güntelberg charging process or by direct force integration, producing

$$
W(z)=\frac{q^{2} e^{-2 \kappa z}}{4 D z} .
$$

The density profile for cations and anions is found by substituting this expression into Eq. (9)

$$
\rho_{ \pm}(z)=\rho \exp \left(\frac{b e^{-2 \kappa z}}{2 z}\right) .
$$

We have defined $b$ as half the Bjerrum length, $b=\lambda_{B} / 2$ $=q^{2} / 2 D k_{B} T$. For water at room temperature $b \approx 3.6 \AA$ - comparable to the size of a hydrated ion-and the density profile rapidly reaches its bulk value. The amount of solute adsorbed can now be calculated by inserting Eq. (17) into Eq. (1). With skillful complex variable analysis, OS were able to integrate the Gibbs adsorption isotherm, obtaining an expression for the excess surface tension as an infinite series in $\kappa b .{ }^{9}$ As was discussed in Sec. II, this procedure does not conserve the number of particles in the system and is, intrinsically, grand canonical. We now present an alternative, canonical, calculation of the excess surface tension.

Let us suppose that the electrolyte is confined to a cylinder of height $H$ and cross-sectional area $A$. If the interfaceion interactions are neglected (periodic boundary condi- tions), the electrolyte will be uniformly distributed over the volume of the cylinder. In the thermodynamic limit, the electrostatic free energy can be easily calculated from the Debye-Hückel theory, yielding

$$
F^{\text {bulk }}=-\frac{q^{2} \kappa}{3 D} N_{t}
$$

where $N_{t}=N_{+}+N_{-}$is the total number of solute particles. On the other hand, the presence of an interface produces a concentration gradient characterized by the normalized distribution

$$
\rho_{ \pm}(z)=\frac{N_{ \pm} e^{-\beta W(z)}}{A \int_{0}^{H} e^{-\beta W(z)} d z} .
$$

Now, suppose we fix an ion some distance $z$ from the interface, far from the radial boundary of the cylinder. The electrostatic potential in the vicinity of this ion is approximately given by Eq. (15) — where, in view of the thermodynamic limit of Eq. (8), we can neglect the finite size corrections. Evidently this potential is produced by the ion itself, as well as by the interface and the ionic atmosphere. The potential that the ion feels due to polarization of the interface and the ionic atmosphere is

$$
\psi_{0}(z)=\lim _{\substack{r_{1} \rightarrow 0 \\ r_{2} \rightarrow 2 z}}\left(\psi\left(r_{1}, r_{2}\right)-\frac{q}{D r_{1}}\right)=\frac{-q \kappa}{D}+\frac{q e^{-2 \kappa z}}{2 D z} .
$$

The electrostatic energy is

$$
E=\frac{A q}{2} \int_{0}^{H}\left(\rho_{+}(z)+\rho_{-}(z)\right) \psi_{0}(z) d z .
$$

This can be subdivided into the bulk and surface contributions, corresponding to the first and the second term of Eq. (20), respectively. The bulk term is easily integrated, yielding

$$
E^{b}=\frac{-q^{2} \kappa}{2 D} N_{t} .
$$

The surface contribution is found to be

$$
E^{s}=\frac{q^{2} N_{t}}{4 D} \frac{\int_{0}^{H} \exp (-2 \kappa z-\beta W(z)) \frac{d z}{z}}{\int_{0}^{H} \exp (-\beta W(z)) d z} .
$$

Now, in the limit $H \rightarrow \infty$ relevant for the calculation of surface tension

$$
\lim _{H \rightarrow \infty} \frac{1}{H} \int_{0}^{H}\left(e^{-\beta W(z)}-1\right) d z=0,
$$

and Eq. (23) simplifies to

$$
E^{s}=\frac{A q^{2} \rho}{2 D} \int_{0}^{\infty} e^{-2 \kappa z-\beta W(z)} \frac{d z}{z} .
$$

Unfortunately, no method is available for analytic evaluation of this integral. We note, however, that if the adsorption potential is replaced by its value at infinite dilution, $W(z)$ $\rightarrow W_{\infty}(z)$, the integral can be done explicitly, yielding 


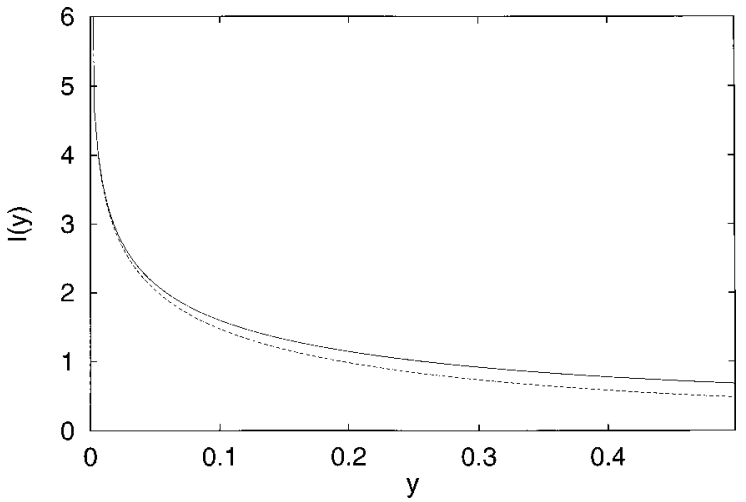

FIG. 1. The solid curve is the numerically evaluated integral $I(y)$, as a function of $y=\kappa b$, Eq. (27); the dashed curve is the analytic expression obtained when $W(z) \rightarrow W_{\infty}(z)$; see Eq. (26).

$$
E^{s}=\frac{q^{2} \rho A}{D} K_{0}(2 \sqrt{b \kappa})
$$

where $K_{\nu}(x)$ is the modified Bessel function of order $\nu$. Replacement of the adsorption potential by its value at infinite dilution should be a good approximation, since the large distances-for which the discrepancy between $W(z)$ and $W_{\infty}(z)$ becomes significant-are not important because of the exponential drop in the electrostatic potential away from the interface. To confirm this, we have numerically evaluated the integral

$$
I(\kappa b)=\int_{0}^{\infty} e^{-2 \kappa z-\beta W(z)} \frac{d z}{z},
$$

and compared it with the exact analytic expression obtained when $W(z) \rightarrow W_{\infty}(z)$, Fig. 1 . As was hoped, the agreement is indeed quite good, extending all the way to $y \equiv \kappa b \approx 0.45$. In water at room temperature, this corresponds to concentration of $0.15 \mathrm{M}$, which is above the maximum for which the limiting laws of this paper can be expected to apply realistically.

The electrostatic free energy can now be obtained through the Debye charging process, in which all the particles are simultaneously charged from zero to their full charge $e^{3,10}$

$$
F=\int_{0}^{1} 2 E(\lambda q) \frac{d \lambda}{\lambda} .
$$

Note that both $b$ and $\kappa$ are dependent on $q$, so that $b(\lambda q)$ $=\lambda^{2} b(q)$ and $\kappa(\lambda q)=\lambda \kappa(q)$. The integral can once again be done explicitly, yielding the electrostatic free energy of an electrolyte solution in the presence of an interface

$$
\begin{aligned}
F= & -\frac{q^{2} \kappa}{3 D} N_{t}-\frac{A q^{2} \rho}{2 D}\left[2 K_{0}(2 \sqrt{y}){ }_{1} F_{2}(1 ; 2 / 3,5 / 3 ; y)\right. \\
& \left.+3 \sqrt{y} K_{1}(2 \sqrt{y}){ }_{1} F_{2}(1 ; 5 / 3,5 / 3 ; y)\right] .
\end{aligned}
$$

Here, ${ }_{p} F_{q}$ is the generalized hypergeometric function ${ }^{14}$ and $y \equiv b \kappa$. Substituting Eq. (29) into Eq. (8), the increase in surface tension of water due to 1:1 electrolyte is,

$$
\begin{aligned}
\sigma^{e x}= & \sigma_{0}^{e x}\left[2 K_{0}(2 \sqrt{y}){ }_{1} F_{2}(1 ; 2 / 3,5 / 3 ; y)\right. \\
& \left.+3 \sqrt{y} K_{1}(2 \sqrt{y}){ }_{1} F_{2}(1 ; 5 / 3,5 / 3 ; y)\right],
\end{aligned}
$$

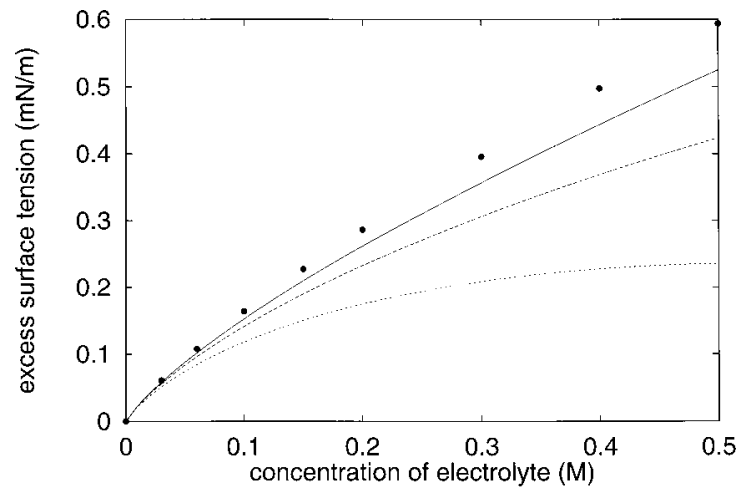

FIG. 2. The excess surface tension of an aqueous solution of a 1:1 electrolyte at room temperature. The solid curve is the analytic expression given by Eq. (30); the long dashed curve is the sum of the first 20 terms of the OS result (Ref. 9); the short dashed curve is the Onsager-Samaras limiting law, Eq. (31); the solid points are the result of numerical integration of Eq. (28) with full $W(z)$.

where $\sigma_{0}^{e x}=q^{2} \rho / 2 D$. For very low concentrations, this expression reduces to the limiting law found by OS

$$
\sigma_{l}^{e x}=\sigma_{0}^{e x}\left[-\ln (y)-2 \gamma_{E}+3 / 2\right],
$$

where $\gamma_{E}=0.57721566490153 \ldots$ is the Euler's constant.

\section{RESULTS AND DISCUSSION}

We have presented a canonical calculation of the excess surface tension in an electrolyte solution. Unlike the earlier grand-canonical method of Onsager and Samaras, our approach leads to an analytic expression for the excess surface tension expressed in terms of Bessel and hypergeometric functions. It is gratifying, however, that in spite of all the approximations, the two ensembles produce the identical limiting law. This thermodynamic self-consistency suggests that the OS limiting law is, indeed, exact to zeroth order in $D^{\prime} / D$. In Fig. 2 we plot, as a function of concentration, the $\sigma^{e x}$ Eq. (30); the sum of the first 20 terms of the infinite series for the excess surface tension obtained by OS, ${ }^{9}$ and the OS limiting law, Eq. (31). The surface tension $\sigma$ is measured in $\mathrm{mN} \cdot \mathrm{m}^{-1}$ and the concentration of salt $c$ in moles/liter (M) so that

$$
\begin{aligned}
& \sigma_{0}^{e x}=\frac{69.4692 c}{D}\left(\mathrm{mN} \cdot \mathrm{m}^{-1}\right), \\
& y=\frac{4201742 \sqrt{c}}{(D T)^{3 / 2}} .
\end{aligned}
$$

For water at room temperature, $D \approx 78.54$. We note that both ensembles agree fairly well over the full range of concentrations, with the canonical calculation predicting a somewhat larger excess surface tension. In Fig. 1 it was shown that the substitution $W(z) \rightarrow W_{\infty}(z)$ leads to a good approximation for the electrostatic energy. To confirm that this also extends to the free energy, we have performed the Debye charging process, Eq. (28), numerically using the explicit form of $W(z)$, Eq. (16). The result is plotted as solid points in Fig. 2. Indeed, the numerically calculated surface tension agrees quite well with the analytic result, Eq. (30), obtained with the substitution $W(z) \rightarrow W_{\infty}(z)$. 
It has been noted that the OS theory gives a fairly good quantitative description for concentrations up to $0.1 \mathrm{M}$, above which it consistently underestimates the increase in the interfacial tension. ${ }^{5-8}$ The canonical calculation presented above extends the range of agreement between theory and experiment. It is, however, unrealistic to demand that the theory presented above should apply to concentrated solutions, for which even the bulk thermodynamic properties lose their universality. Thus, for concentrations above $0.2 \mathrm{M}$, the molecular nature of the solvent as well as the lyotropic properties of solute will become important. In fact, it has been observed experimentally that for concentrated solutions the excess surface tension increases linearly with the concentration of electrolyte. For an aqueous solution of $\mathrm{NaCl}$ at $T$ $=25^{\circ} \mathrm{C}$ it is found that $\sigma^{e x} \approx 1.6 c,{ }^{15}$ which begins to show strong deviation from Eq. (30) for $c>0.2 \mathrm{M}$.

\section{ACKNOWLEDGMENTS}

This work was supported in part by CNPq-Conselho Nacional de Desenvolvimento Científico e Tecnológico and FINEP_Financiadora de Estudos e Projetos, Brazil.
${ }^{1}$ A. Heydweiller, Ann. Phys. (Leipzig) 33, 145 (1910); Phys. Z. 3, 329 (1902).

${ }^{2}$ C. Wagner, Phys. Z. 25, 474 (1924).

${ }^{3}$ P. W. Debye and E. Hückel, Phys. Z. 24, 185 (1923).

${ }^{4}$ J. W. Gibbs, Collected Works (Longmans, Green and Co., New York, 1928).

${ }^{5}$ F. A. Long and G. C. Nutting, J. Am. Chem. Soc. 64, 2476 (1942).

${ }^{6}$ J. E. B. Randles, in Advances in Electrochemistry and Electrochemical Engineering, edited by P. Delahay and C. W. Tobis (Interscience, New York, 1963), Vol. 3, p. 1.

${ }^{7}$ J. Ralston and T. W. Healy, J. Colloid Interface Sci. 42, 1473 (1973).

${ }^{8}$ P. K. Weissenborn and R. J. Pugh, J. Colloid Interface Sci. 184, 550 (1996).

${ }^{9}$ L. Onsager and N. N. T. Samaras, J. Chem. Phys. 2, 528 (1934).

${ }^{10}$ M. E. Fisher and Y. Levin, Phys. Rev. Lett. 71, 3826 (1993); Y. Levin and M. E. Fisher, Physica A 225, 164 (1996).

${ }^{11}$ V. C. Weiss and W. Schröer, J. Phys.: Condens. Matter 12, 2637 (2000).

${ }^{12}$ E. Güntelberg, Z. Phys. Chem., Stoechiom. Verwandtschaftsl. 123, 199 (1926).

${ }^{13}$ F. H. Stillinger, J. Chem. Phys. 35, 1584 (1961).

${ }^{14}$ W. N. Bailey, Generalized Hypergeometric Functions (Cambridge University Press, London, 1935); L. J. Slater, Generalized Hypergeometric Functions (Cambridge University Press, London, 1966).

${ }^{15}$ N. Matubayasi, H. Matsuo, K. Yamamoto, S. Yamaguchi, and A. Matuzawa, J. Colloid Interface Sci. 209, 398 (1998). 